\title{
PREVALENCE AND EXTENT OF GLYCEMIC EXCURSIONS IN WELL- CONTROLLED PATIENTS WITH TYPE 2 DIABETES MELLITUS USING CONTINUOUS GLUCOSE-MONITORING SYSTEM
}

\author{
SHOKOUFEH BONAKDARAN, REZA RAJABIAN
}

\section{ABSTRACT}

BACKGROUND: Continuous glucose-monitoring system (CGMS) is a tool for assessment of glycemic excursions. Glucose variability is a risk factor independent of glycosylated hemoglobin (HbAlc) for diabetic complications; hence CGMS may be a better method for management of diabetes. AIM: To evaluate the extent of glycemic excursions in wellcontrolled type 2 diabetic patients. SETTING AND DESIGN: The study was carried out in 21 diabetic patients on oral agents. MATERIALS AND METHODS: Patients underwent continuous glucose-monitoring by CGMS for 3 days. Number and duration of glycemic excursions, correlation coefficient (\%) between CGMS and self-monitoring blood glucose (SMBG), mean absolute difference (\%MAD) and complications of CGMS were analyzed. STATISTICAL ANALYSES: The statistical analyses were performed with the use of mean \pm SD, t-test and Mann-Whitney test. RESULTS: The mean age of patients was $51.9 \pm$ 9.7 years. The mean $\mathrm{HbAlc}$ was $6.7 \pm 0.38 \%$. The mean number of glycemic readings was $753.6 \pm 203.5$ times. The correlation coefficient was 0.83 and the MAD was 11.7 $\pm 8.0 \%$, which were considerable. Three (14.2\%) patients experienced, altogether, 9 hypoglycemic events with an average duration of 162 minutes. Twenty $(94.7 \%)$ patients had hyperglycemic events. The mean duration of hyperglycemia was $19.4 \pm 12.8$ hours. All events were asymptomatic. Disconnection of device was the most common complication (3 patients). CONCLUSION: This study demonstrated that well-controlled type 2 diabetic patients have a considerable number of hypoglycemia and hyperglycemia events that may be missed by SMBG.

Key words: Continuous glucose-monitoring system, hyperglycemia, hypoglycemia, self-monitoring blood glucose, type 2 diabetes

DOI: $10.4103 / 0019-5359.49237$

\section{INTRODUCTION}

The benefits of intensive management of diabetes in prevention of chronic complications

Mashhad University of Medical Science, Endocrine

Research Center, Ghaem Hospital, Mashhad, Iran

Correspondence:

Dr. Shokoufeh Bonakdaran, Endocrine Research Center,

Faculty of Medicine, Ghaem Hospital, Mashhad, Iran.

E-mail: bonakdaransh@mums.ac.ir have been established.[1] Frequent selfmonitoring of blood glucose (SMBG) and glycosylated hemoglobin ( $\mathrm{HbA1c}$ ) offered the possibility of control of glycemia, but $\mathrm{HbA1c}$ is a measure for prediction of average glycemia; and despite an ideal $\mathrm{HbA1c}$, several events of glycemia may occur. ${ }^{[2,3]}$ SMBG is a partial and incomplete picture of blood glucose excursions. ${ }^{[4,5]}$ Hypoglycemia, especially 
nocturnal hypoglycemia, is the most prevalent complication in the tight control of diabetes that is usually not detected by SMBG. ${ }^{[6]}$ In addition, postprandial hyperglycemia may occur even in well-controlled type 2 diabetic patients. ${ }^{[7]}$ The role of postprandial hyperglycemia in the pathogenesis of diabetic complications has been confirmed, ${ }^{[8,9]}$ which is usually neglected in patients who are managed by SMBG. The continuous glucose-monitoring system (CGMS) allows identification of glucose excursions. The aim of this study was to determine the prevalence and extent of unrecognized hypoglycemia and hyperglycemia in wellcontrolled diabetic patients.

\section{MATERIALS AND METHODS}

\section{The CGMS system}

The sensor of CGMS is inserted subcutaneously into the tissue of anterior abdomen wall in all patients. ${ }^{[10]}$ All sensors are inserted by one person. The difference in plasma and interstitial fluid glucose was estimated to be $<6 \% .{ }^{[11]}$ This system helps to identify periods of significant glycemic excursions and allows physicians to suggest specific changes in the timing and dosage of drugs or changes in timing and frequency of blood glucose measurement or in dietary regimes of patients. ${ }^{[12]}$ The accuracy of CGMS sensor was based on its correlation with SMBG by Pearson correlation during hypoglycemia and hyperglycemic events, with significant $P$ values $<0.05$.

\section{Patient selection}

Twenty-four type 2 diabetic patients were recruited from among the outpatients. We had a limitation on the sample size because the sensor of CGMS was very expensive; hence we selected a convenient sample size. Patients were eligible for enrollment in the study if they met the following inclusion criteria: diagnosis of type 2 diabetes, oral agent treatment for control of diabetes, an $\mathrm{HbA} 1 \mathrm{C}$ less than $7 \%$ and fasting blood sugar less than $130 \mathrm{mg} / \mathrm{dL}$. It seems that diabetes was well controlled in these patients. The patients were maintained on the diet prescribed by the dietitian, which included isocaloric or hypocaloric diet depending on whether the body mass index (BMI) was $<25$ or $>25 \mathrm{~kg} / \mathrm{m}^{2}$. This diet contained $45 \%$ to $50 \%$ of carbohydrates, $30 \%$ to $35 \%$ of fats and $15 \%$ to $20 \%$ of proteins. The main exclusion criteria included the following: patients with history of insulin treatment, renal failure, liver dysfunction; and those using concomitant drugs that cause hyperglycemia or hypoglycemia. The patients were divided into 2 groups: one group comprising of patients on sulfonylurea or glinids treatment; and the other group comprising of patients on metformin therapy only.

Study protocol: The study was a pilot, descriptive, open-label, non-interventional study. All patients gave informed voluntary consent, and the protocol was approved by the local ethics committee in accordance with the ethical standards of Helsinki Declaration. Patients that satisfied inclusion criteria received intensive training for duration of approximately 1 hour in the use of CGMS. A sensor was then inserted into the abdomen in the subcutaneous tissue. Each patient was asked to record 4 capillary glucose measurements, which were taken each day by using an AcuuCheck glucometer that was provided by us, and then capillary values were entered into the monitor 
for calibration. Also, it was mandatory for all patients to record their dietary programs, the time of all meals, the quality and quantity of meals and the time and duration of any exercise. The timings of meals and drug usage and exercise were entered into the device by the patients. Each patient was asked to refer back to us in 72 hours for disconnection of sensor and download of the data using the Medtronic MiniMed software. We defined a biochemical hypoglycemic event as a glucose value less than $50 \mathrm{mg} / \mathrm{dL}$ with or without symptoms. Postprandial hyperglycemia was defined as a value more than $140 \mathrm{mg} / \mathrm{dL}, 2$ hours after the start of meal. We counted the number of glucose periods with a glucose value less than $50 \mathrm{mg} / \mathrm{dL}$ and their duration, as well as prolonged periods with a glucose value more than $140 \mathrm{mg} / \mathrm{dL}$ postprandial. The correlation coefficient and mean absolute difference (MAD) were calculated by Medtronic software and defined as $>0.79$ and $<28 \%$, respectively, optimal. The MAD was determined by the average value of differences between sensor glucose values and blood glucose values in percentage.

\section{Statistical analyses}

All statistical analyses were performed using the SPSS (statistical package for the social sciences, version 14, SPSS Inc., Chicago, USA) software. Normally distributed quantitative variables were demonstrated as mean \pm standard deviation; and for values which were not normally distributed, median and the interquartile range were used. For comparison of quantitative variables, the independent $t$ test and Mann-Whitney test were utilized. Pearson correlation was used to compare sensor readings with SMBG results.

\section{RESULTS}

Twenty-four patients with type 2 diabetes mellitus participated in this study. In 3 patients, CGMS was disconnected and they were excluded from the study. The baseline characteristics of 21 patients are shown in Table 1. The mean number of glucose readings by CGMS during the 72 hours was $753.6 \pm 203.5$ in each patient. This number $(86.7 \%)$ was in the normal range (normal, greater than $80 \%$ ). The correlation coefficient between SMBG and CGMS was 0.83 (normal, greater than 0.79 ). The mean value of MAD was $11.7 \pm 8.0 \%$ (normal, less than $28 \%$ ). The mean glucose values were as follows: $125.6 \pm 20.5 \mathrm{mg} / \mathrm{dL}$ in fasting state; $156.6 \pm$ $54.1 \mathrm{mg} / \mathrm{dL}, 2$ hours post-breakfast; $141.8 \pm$ $24.6 \mathrm{mg} / \mathrm{dL}, 2$ hours post-lunch; $135.0 \pm 25.9$ $\mathrm{mg} / \mathrm{dL}, 2$ hours post-dinner. During the study, $3(14.2 \%)$ patients experienced a total of 9 hypoglycemic events with average duration of 162 minutes (range, $45-378 \mathrm{~min}$ ). Seven $(77.8 \%)$ of the hypoglycemic events occurred between $10 \mathrm{pm}$ and $4 \mathrm{am}$. All the events were asymptomatic. We divided the patients into 2 groups: group I, comprising of patients whose treatment regimens included only metformin; and group II, comprising of patients who

Table 1: Clinical and biochemical data of the study population

\begin{tabular}{lc}
\hline Age (years) & $51.04 \pm 9.7$ \\
$12(57.1 \%)$ male, & Sex (number, \%) \\
$9(42.9 \%)$ female & \\
Duration of diabetes (month) & $24.0(24-60)^{*}$ \\
Body mass index (kg/m2) & $26.9 \pm 2.7$ \\
Systolic blood pressure(mm Hg) & $123.5 \pm 14.3$ \\
Diastolic blood pressure(mm Hg) & $77.1 \pm 8.7$ \\
HbA1C (\%) & $6.7 \pm 0.38$ \\
Fasting blood sugar(mg/dl) & $115.6 \pm 10.5$ \\
Ischemic heart disease (number, \%) & $9(19 \%)$ \\
Retinopathy (number, \%) & $1(4.8 \%)$ \\
Albuminuria (number, \%) & $2(9.5 \%)$
\end{tabular}

*Median with (interquartile) 
received sulfonylurea or meglitinids in their treatment regimen. No significant difference was found between the 2 groups in duration $(P$ $=0.08, \mathrm{df}=19)$ and the number of hypoglycemia events $(P=0.11, \mathrm{df}=19)$. Another important observation in this study was the detection of postprandial hyperglycemia. Twenty (94.7\%) patients had hyperglycemic events. Mostly, these events occurred after breakfast. The mean number of hyperglycemic events was 8.8 \pm 4.5 times in each patient with a mean duration of $19.4 \pm 12.8$ hours (minimum, 35 minutes; and maximum, 303 minutes). No significant difference was found in frequency $(P=0.92$ with $\mathrm{df}=19$, mean difference $=0.24,95 \% \mathrm{Cl}=$ -4.9 to 5.4$)$ and duration of hyperglycemia $(P=$ .83 with $\mathrm{df}=19$, mean difference $=-1.2,95 \%$ $\mathrm{Cl}=-13.8$ to 11.3 ) between the 2 groups. The characteristics of patients according to groups

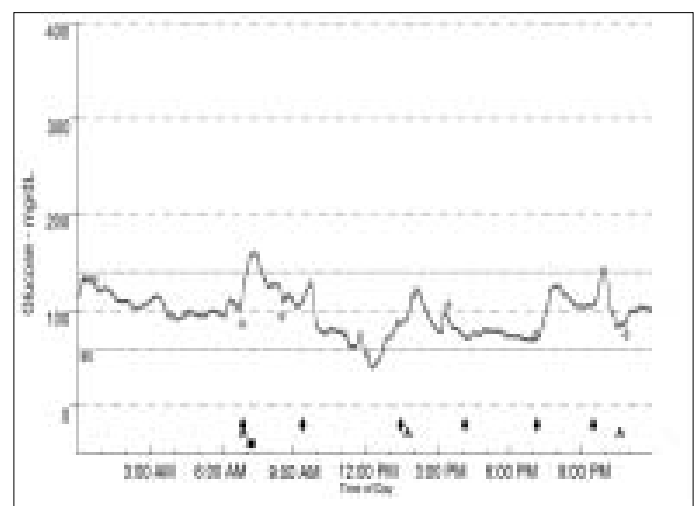

Figure 1: Twenty-four glucose profiles from a patient on metformin therapy demonstrating hypoglycemic and hyperglycemic events are shown in Table 2. A typical 24-hour sensor tracing of a patient with metformin treatment and a tracing of a patient on glibenclamide therapy are shown in Figures 1, 2, respectively. We analyzed all complications during sensor implantation (bleeding, pain) and during the exam (local infection, disconnection [technical problem]). Disconnection was the most common complication in $3(12.5 \%)$ of the patients. No trauma, local infection, allergy, bleeding or other complications were observed in our patients. All patients (with the exclusion of the patients in whom the device got disconnected) completed the CGMS integrity.

\section{DISCUSSION}

The main finding was the high frequency of postprandial hyperglycemia in well-controlled

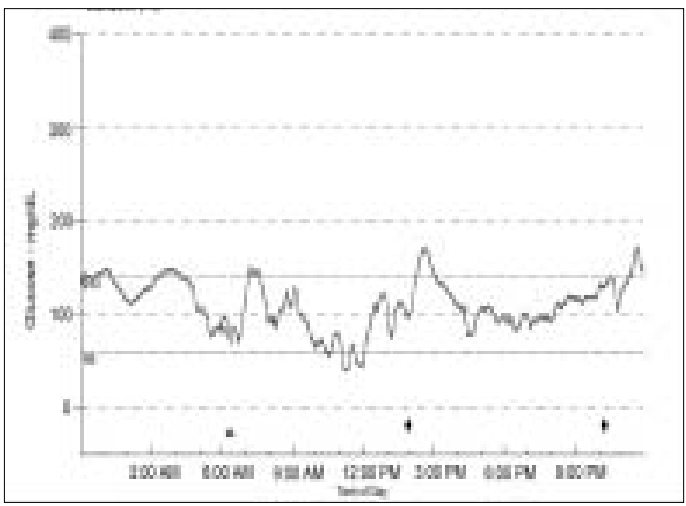

Figure 2: Twenty-four glucose profiles from a patient on glibenclamide demonstrating hypoglycemic and hyperglycemic events

Table 2: Characteristics of the study population according to type of treatment

\begin{tabular}{lccc}
\hline Number of patients & Only metformin & $\begin{array}{c}\text { Metformin + sulfonylurea or } \\
\text { only sulfonylurea }\end{array}$ & $P$ \\
Number of hyperglycemic event & $12(57.1 \%)$ & $9(42.8 \%)$ & 0.92 \\
Duration of hyperglycemia (hour) & $8.9 \pm 5.4$ & $9.1 \pm 4.1$ & 0.83 \\
Number of hypoglycemic event & $19.1 \pm 10.7$ & $17.9 \pm 13.6$ & 0.11 \\
Duration of hypoglycemia (hour) & $0.1 \pm 0.3$ & $1.2 \pm 2.5$ & 0.08 \\
\hline
\end{tabular}


patients. Postprandial hyperglycemia has been shown to be closely related with cardiovascular disease, ${ }^{[13]}$ aggravation of oxidative stress and endothelial dysfunction. ${ }^{[14,15]}$ The high frequency of hyperglycemic events in our study is consistent with other studies ${ }^{[7,9,16]}$ and indicates that relying on only $\mathrm{HbA1c}$ in the management of diabetes (as is being advised presently) is inadequate for reducing the rate of cardiovascular complications. Another primary outcome of our study was to detect hypoglycemia in well-controlled patients. Nocturnal hypoglycemia is a problem specific to tightly controlled diabetic patients, which can lead to delays in correction of the hypoglycemia. In ADVANCE study, severe hypoglycemia occurred in more patients in the intensively treated arm $(2.7 \%$ versus $1.5 \%)$. In one study by Hay $\mathrm{HC}$ et al. on old patients with well-controlled type 2 diabetes, it was shown that $80 \%$ of patients experienced hypoglycemia. ${ }^{[7]} \mathrm{We}$ found that $14.2 \%$ of our patients had hypoglycemic events. The frequency is less than that in previous studies. We think this is due to the age group of our patients being different when compared to previous studies. In our results, no significant difference was found in hypoglycemic and hyperglycemic events between patients with only metformin usage and those on other treatments. Iatrogenic hypoglycemia with metformin was less frequent than with sulfonylurea, but it has been reported in other studies. ${ }^{[17,18]}$ These results may be related to the small sample size in the 2 groups of patients. Analyses of initial multicenter evaluation of CGMS revealed a median correlation between sensor and SMBG readings 0.92. ${ }^{[19]}$ Despite the difficulty of wearing CGMS for 72 hours, it was well tolerated by most patients. The most common complication was disconnection of device, which is similar to other studies..$^{[4,20]}$ Other complications were not registered in our study and in other studies. This study is the first report on utility of CGMS and evaluation of glycemic excursions in well-controlled type 2 diabetes patients in Iran. The most important limiting factor in the interpretation of our results was the small sample size. A recent metaanalysis for investigation of potential effects of CGMS in type 1 diabetes found that CGMS is not better than SMBG in improving metabolic control, ${ }^{[21]}$ but all trials in type 2 diabetes are small. Therefore, our results cannot be generalized to all type 2 diabetic patients. More well-conducted clinical studies with larger number of participants are needed.

\section{ACKNOWLEDGMENT}

We would like to thank MUMS Research Council for financial support; and Parsian Diabetic Center for referring patients.

\section{REFERENCES}

1. Stratton IM, Adler Al, Neil HA, Matthews DR, Manley SE, Cull CA, et al. Association of glycaemia with macrovascular and microvascular complications of type 2 diabetes (UKPDS 35): Prospective observational study. BMJ 2000;321:405-12.

2. Rohlfing $\mathrm{CL}$, Wiedmeyer HM, Little RR, England JD, Teniil A, Goldstein DE. Defining the relationship between plasma glucose and $\mathrm{HbA1C}$ : Analysis of glucose profiles and $\mathrm{HbA1C}$ in the Diabetes Control and Complication Trial. Diabetes Care 2002;25:275-8.

3. Kohnert KD, Augstein P, Heike P, Zander E, Peterson K, Freyse EJ, et al. Chronic hyperglycemia but not glucose variability 
determines $\mathrm{HbA} 1 \mathrm{C}$ levels in well- controlled patients with type 2 diabetes. Diabetes Res Clin Pract 2007;77:420-6.

4. Boland E, Mansod T, Delucia M, Brandt CA, Fernando S, Tamborlane WV. Limitation of Conventional methods of self monitoring of blood glucose. Diabetes Care 2001;24:1858-62.

5. Hirsch IB, Brownlee M. Should minimal blood glucose variability become gold standard of glycemic control? J Diabetes Compl 2005;19: 178-81.

6. Cryer PE, Davis SN, Shamoon H. Hypoglycemia in diabetes. Diabetes Care 2003;26:1902-12.

7. Hay LC, Wilmshurst EG, Flucher G.Unrecognized hypo and hyperglycemia in well Controlled patients with type 2 diabetes mellitus. Diabetes Technol Ther 2003;5:19-26.

8. Ceriello A. The postprandial state and cardiovascular disease: Relevance to diabetes mellitus. Diabetes Metab Rev 2000;16:125-32.

9. Hanefeld M. Postprandial hyperglycemia: Noxious effects on the vessel wall. Int J Clin Prac 2002;129:45-50.

10. Ionescu-Tirgoviste C, Guja C, loacara S, Dumitrescu D, Tomescu I. Continuous Glucose monitoring: Physiologic and pathophysiologic significance. Rom J Intern Med 2004;42:381-93.

11. Rebrin K, Steil GM. Can interstitial glucose assessment replaces blood glucose measurement? Diabetes Technol Ther 2000;2:461-72.

12. Sachedina N, Pickup JC. Performance assessment of the Medtronic-MiniMed Continuous Glucose Monitoring System and its use for measurement of glycaemic control in Type 1 diabetic subjects. Diabetes Med 2003;20:1012-5.

13. Cavalot F, Petrelli A, Travers M, Bonomo K, Fiora $\mathrm{E}$, Conti $\mathrm{M}$, et al. Postprandial blood glucose is a stronger predictor of cardiovascular events than fasting blood glucose in type 2 diabetes mellitus, particular in women. J Endocrinol Metab 2006;91:813-9.

14. Ceriello A, Cavarape A, Martinelli L, Da Ros R, Marra G, Quagliaro L, et al. The postprandial state in type 2 diabetes and endothelial dysfunction: Effects of insulin aspart. Diab Med 2004;21:171-5.

15. Bonora E. Postprandial peaks as a risk factor for cardiovascular disease: Epidemiological perspectives. Int J Clin Pract 2002;129:5-11.

16. Bode BW, Schwartz S, Stubbs HA, Block JE. Glycemic characteristics in continuously monitored patients with type 1 and type 2 diabetes: Normative values. Diabetes Care 2005;28:2361-6.

17. The United Kingdom Prospective Diabetes Study group. Effect of intensive blood- glucose control with metformin on complications in overweight patients with type 2 diabetes. Lancet 1998;352:854-65.

18. The United Kingdom Prospective Diabetes Study Research Group. Overview of 6 years of therapy type II diabetes: A progressive disease. Diabetes 1995;44:1249-58.

19. Mastrototaro J. The MiniMED Continuous Glucose Monitoring System (CGMS). J Pediatr Endocrinol Metab 1999;12:751-8.

20. Djakoure -Platonoff C, Radermercker R, Reach G, Slama G, Slama JI. Accuracy of the continuous glucose monitoring system in inpatient and outpatient condition. Diabetes Metab 2003;29:159-62.

21. Golicki DT, Golicka D, Groele L, Pankowska E. Continuous Glucose Monitoring System in children with type 1 diabetes mellitus: A systemic review and meta- analysis. Diabetologia 2008;51:233-240.

Source of Support: MUMS Research Council, Conflict of Interest: None declared. 